\title{
Characterisation of diamond abrasive grains of grinding tools using industrial X-ray computed tomography
}

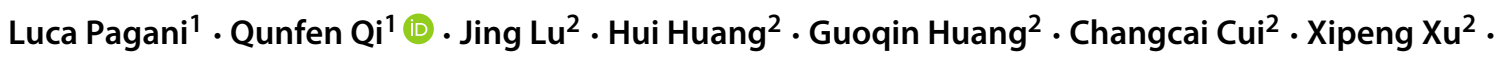 \\ Xiangqian Jiang ${ }^{1}$
}

Received: 26 March 2020 / Accepted: 12 October 2020 / Published online: 15 November 2020

(C) The Author(s) 2020

\begin{abstract}
In this paper, a characterisation of diamond abrasive grains of grinding tools using industrial X-ray computed tomography (XCT) is carried out. One of the most challenge tasks in the characterisation is extracting the diamond abrasive grains from the XCT volume data. Methods that are able to extract the grains are then developed and introduced in this paper. The first step is to create a triangular mesh surface from the reconstructed volume file using a gradient anisotropic diffusion filter. The second step is to convert the measured greyscale volume into a signed distance field using a global threshold value and then a localised method for grain segmentation. To validate the proposed method, three different types of grinding tool specimens are measured and analysed. Each abrasive grain is segmented and the distributions of grains (with both random and designed patterns) are then calculated, plotted and analysed. The quantitative analysis clearly shows the deviations between the measured distribution and the designed pattern of the grinding tool, which indicates that the proposed method can provide an accurate and comprehensive characterisation of the grinding tools.
\end{abstract}

Keywords Industrial X-ray computed tomography · 3D characterisation · Grinding tool · Grinding wheel · Sol-gel ball

\section{Introduction}

Characterisation of grinding tool plays a vital role in ensuring the precision of grinding process and on the surface topography produced. Generally, the characterisation has been largely focused on the tool's topography. As most of the grinding tools are rough, random, multi-scale and multi-material, it is not an easy task to extract their surface topography via widely available contact/non-contact measurement instruments. Stylus-based contact measurement includes profilometers and coordinate measuring machines (CMMs) can be used to measure surface topography, form and dimensions of a grinding tool with high accuracy. They are generally slower and can collect a lower number of measurement points on the surface. Their stylus can be easily worn on the course of a trace on the wheel surface, which will subsequently distort the measured result. To avoid such distortion, various of non-contact measurement techniques

Qunfen Qi

q.qi@hud.ac.uk

Extended author information available on the last page of the article. have been used, including laser triangulation system [1], scanning laser microscope (SLM) [2, 3], binocular vision system [4], laser microscope [5], white light interferometry (WLI) [6, 7], and focus-variation technology [8].

Nevertheless, both the contact and non-contact measurement instruments cannot directly separate abrasive grains from the grinding tool, which is of uttermost importance to the performance of the tool. To extract abrasive topography from the measurement data, specific algorithms have to be developed. This will often include a sequence of operations, such as filtration to remove data that is out the scope of the analysis, and then matching and extracting [6,9]. These studies are mainly based on the characterisation of surface topography, in which topography features such as peaks and dales are the focus of the analyses, but abrasive grains' topography can hardly be separated correctly from the bonding material.

As part of the non-contact measurement group, industrial $\mathrm{X}$-ray computed tomography (XCT) technology, in recent year, has started as a popular measurement method for geometrical characterisation [10]. Industrial X-ray CT technology is able to measure as well as the inner as the outer geometry of a component in a non-destructive way. Most importantly, it also simultaneously allows performing 


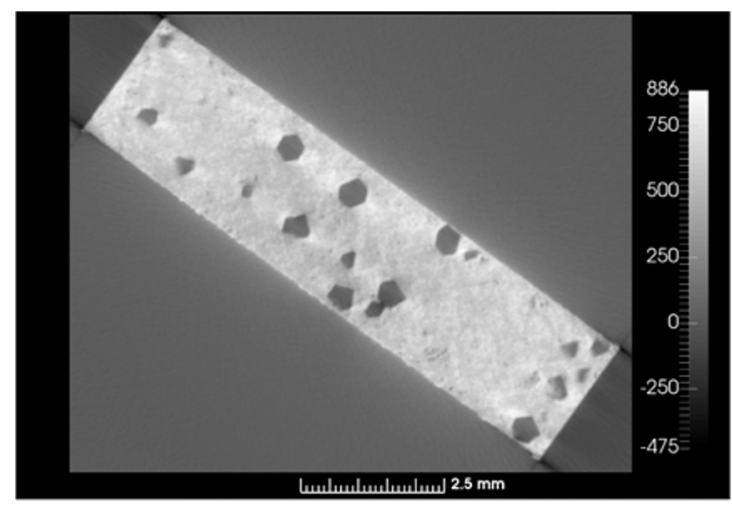

(a) Reconstructed slice

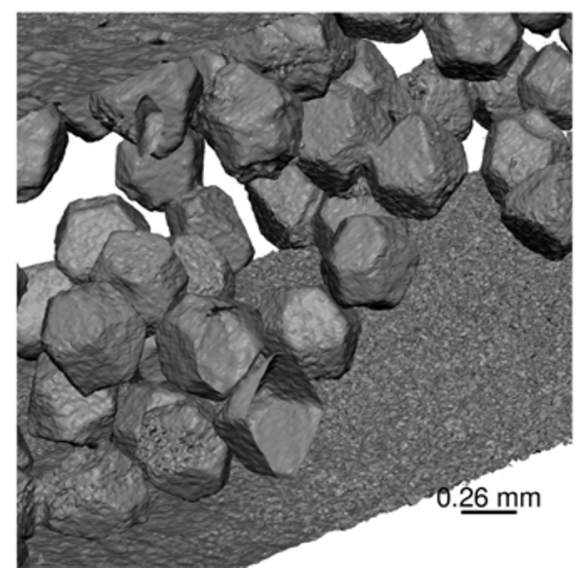

(c) Global threshold surface using the re-( constructed volume

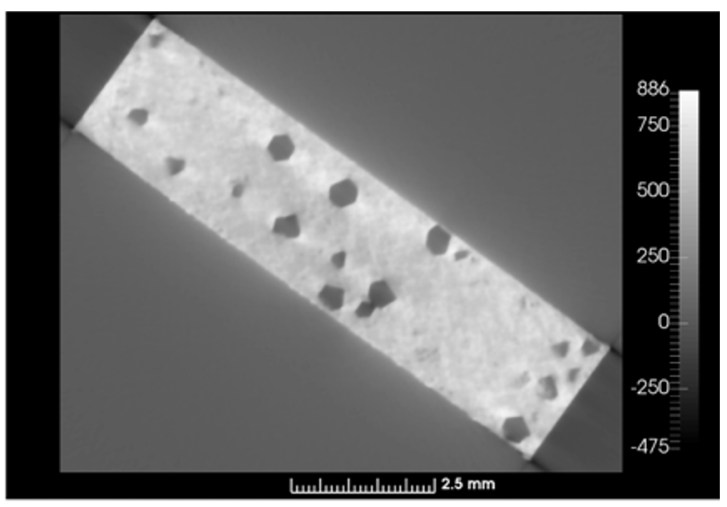

(b) Smoothed slice

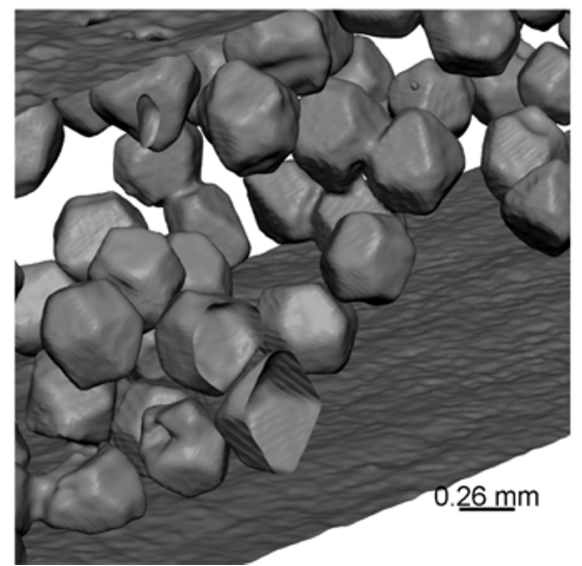

smoothed volume

Fig. 1 Reconstructed and smoothed volumes and extracted iso-surfaces. The colour represents the grey value of the reconstructed XCT volumes

geometrical and material quality assessment with only one measurement process. For example, XCT measurement has been used to assess geometrical variability [11], also to detect flaws such as voids [12] and cracks and to deliver particle analysis in materials [13].

Thanks to the ability of assessing material quality, diamond abrasive grains in the grinding tool can be easily distinguished from the bonding material in the CT measured data. Such characterisation allows not only assessment of every abrasive grain on both the surface and internal of the tool but also distribution of all grains throughout the whole tool, enabling a true 3D characterisation of the tool, which is substantially distinct from the classic methods based on 2.5D surfaces usually measured with optical and stylusbased instruments. Though this technology provides great potential, methods of how to extract the abrasive grains from the reconstructed XCT volume data still stay unstudied.

To this end, a characterisation of diamond abrasive grains of grinding tools using industrial XCT is carried out in this paper. Methods of how to extract the abrasive grains are then studied. Three different types of grinding tool specimens, including a set of segment samples of diamond grinding wheel, two types of sol-gel balls for polishing pad, and a set of diamond grinding discs with designed patterns, are measured and analysed using the proposed method. The paper is constructed as follows. Measurement procedures and the techniques used to reconstruct the abrasive grains' surfaces are introduced in Section 2. Three different types of grinding tools were measured and analysed in Section 3. Section 4 concludes the paper.

\section{Measurement}

$\mathrm{XCT}$ operates by using an X-rayv generator propagate through the object material. As the X-rays are attenuated due to absorption or scattering, an X-ray detector is positioned on the opposite side of the X-ray source to measure the attenuation. This allows XCT to detect the presence of material, especially in the case of multi-material objects. While the measured object rotates over $360^{\circ}$, a set of raw projection data, which is called sinogram, is 


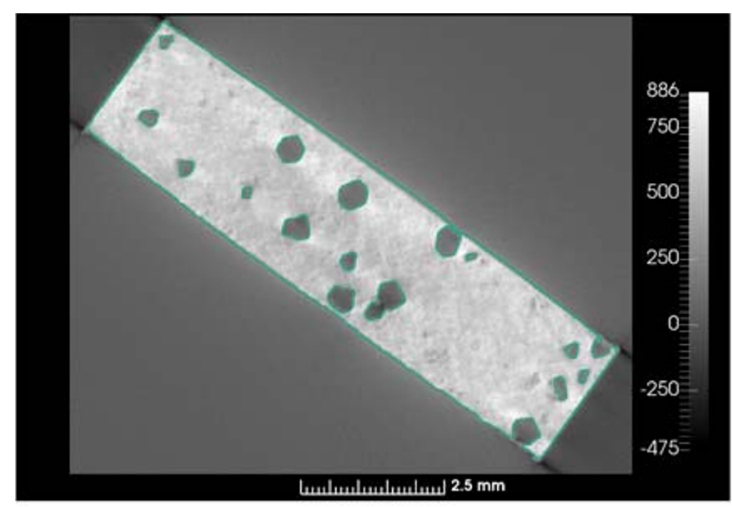

(a) Volume: global threshold

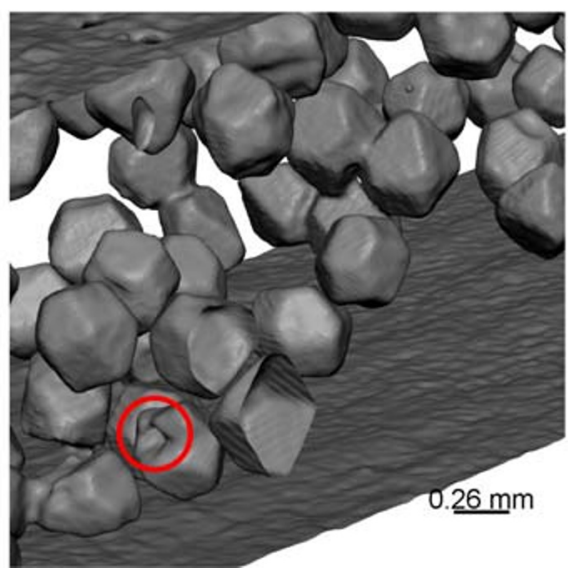

(c) Mesh: global threshold

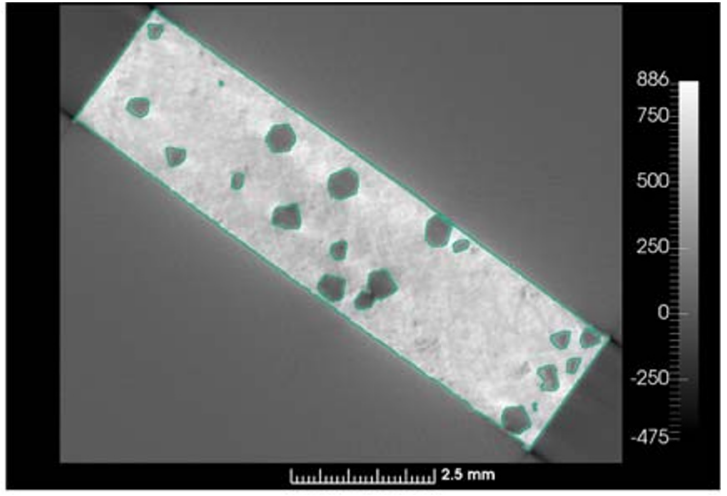

(b) Volume: localised Chan and Vese method

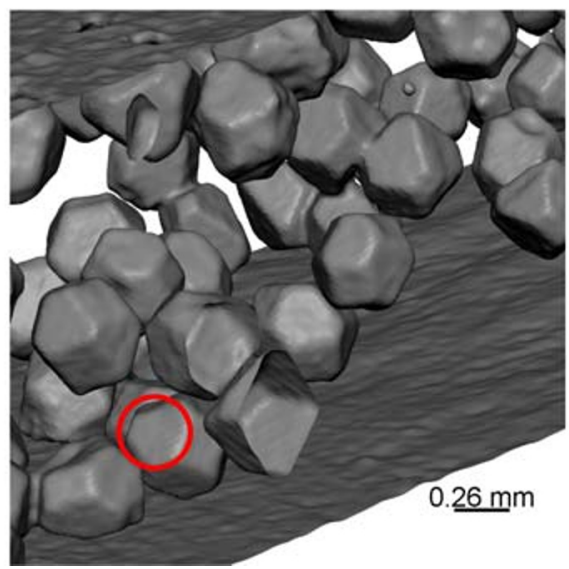

(d) Mesh: localised Chan and Vese method

Fig. 2 Initial segmentation using a global threshold and after applying the localised version of the Chan and Vese algorithm. The colour represents the grey value of the reconstructed CT volumes

obtained and then processed using a form of tomographic reconstruction to generate a series of cross-sectional images. The projection images can then be reconstructed using a specific software into a volume data.

In this study, the device which was used for XCT measurement was X-Tek XTH225kv system (Nikon Metrology). The system offers a $225-\mathrm{kV}$ microfocus $\mathrm{X}$-ray source with a $3-\mu \mathrm{m}$ focal spot size, a large inspection volume, high image resolution and fast CT reconstruction [14]. After the acquisition, the projections were then reconstructed using a commercial software CT Pro 3D [15].

\subsection{Surface determination}

As the reconstructed volume file does not automatically come with a surface, it needs to be processed to create a triangular mesh surface. To determine a triangular mesh surface, a suitable algorithm has to be used to segment different objects (called object segmentation), and then a proper method should be applied to divide each single abrasive grain from other grains and the bonding material.

Before performing the object segmentation, the volume has to be smoothed to allow a better detection of the edges. In this smoothing process, 15 iterations of the gradient anisotropic diffusion filter which was implemented in ITK [16] were used, and the conductance parameter was set to 5 . The conductance parameter was chosen in the range suggested by Mirebeau et al. [17], while the number of iterations was experimentally chosen. It was observed that between 10 and 20 iterations, given the chosen conductance parameter, the quality of the reconstructed surface was acceptable. Figure 1 shows a slice of the volume after the reconstruction and then after applying the smoothing algorithm, where Fig. 1b shows a smoothed version with enhanced edges and high-frequency noise has been removed. The reconstructed iso-surfaces are shown in Fig. $1 \mathrm{c}$ and $\mathrm{d}$ for the raw and the smoothed volume, respectively, which clearly show that the used smoothing technique allowed us to remove the measurement noise. 
Fig. 3 Segmentation performed before and after applying the watershed transform. The color bar represents the grey value of the reconstructed XCT volume, and each colour in (a) and (a) represents a segmented region

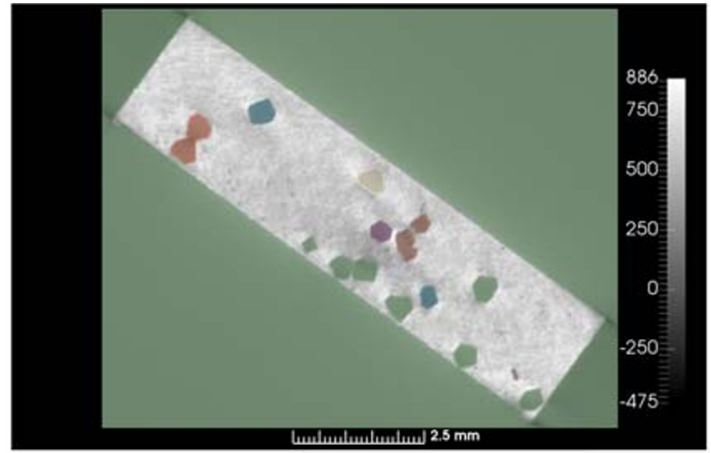

(a) Segmentation without dividing the abrasive grains

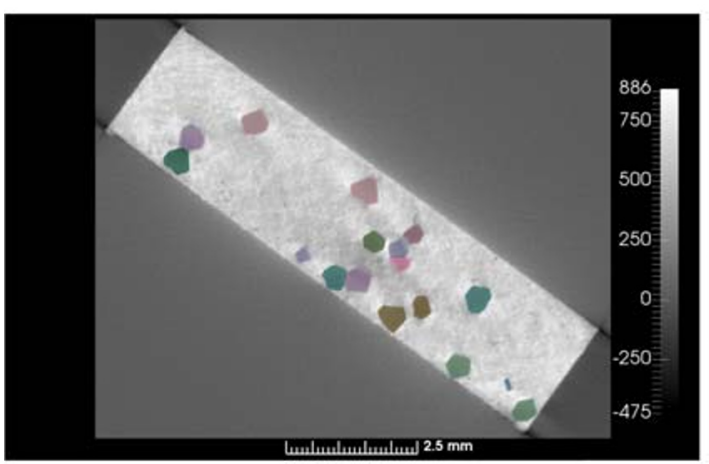

(b) Segmentation after applying the watershed segmentation

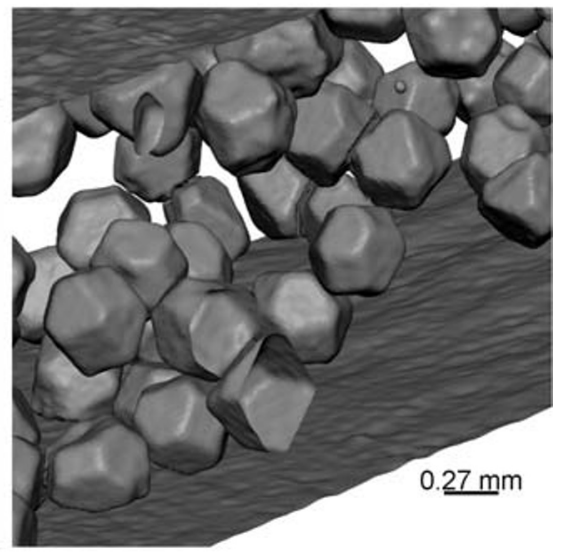

(c) Mesh reconstructed after setting the signed distance on the watershed line to 0.5 voxels

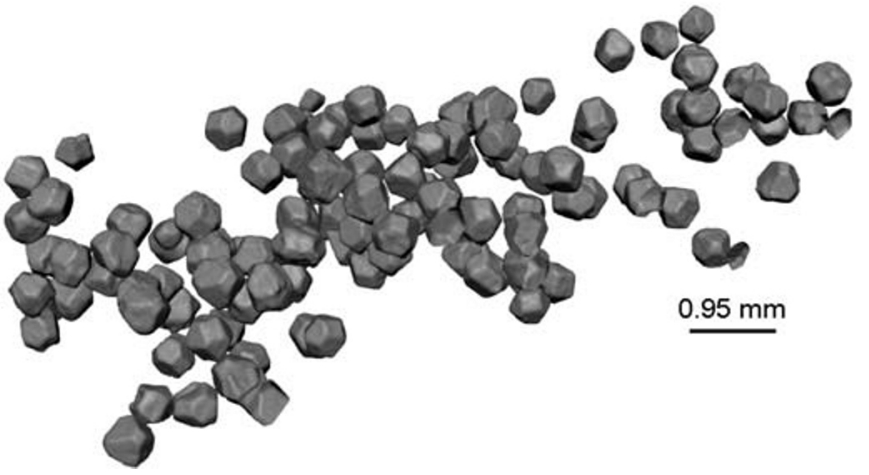

(d) Segmented grains

To perform the object segmentation, the measured greyscale volume is first converted into a signed distance field using a global threshold value. A signed distance field is a function that assign, for each point in the space, the value of the signed distance between a point and the surface. It is defined as

$\phi(x)= \begin{cases}-d(x, \partial \Omega), & \text { if } x \in \Omega \\ d(x, \partial \Omega), & \text { if } x \notin \Omega\end{cases}$ where $\boldsymbol{x}=(x, y, z)^{T}$ is a coordinate in the space, $\Omega$ represents the volume occupied by an object, $\partial \Omega$ is its boundary (surface) and $d(\boldsymbol{x}, \partial \Omega)$ is a distance between a point in the space and a surface. The surface, represented by the isocontour (also called level set) of level zero, is then evolved according to a dynamic model modifying the values of the signed distance field. Many models are available to perform the evolution of the surface in order to optimally segment the surface. They can be divided into two main classes: 


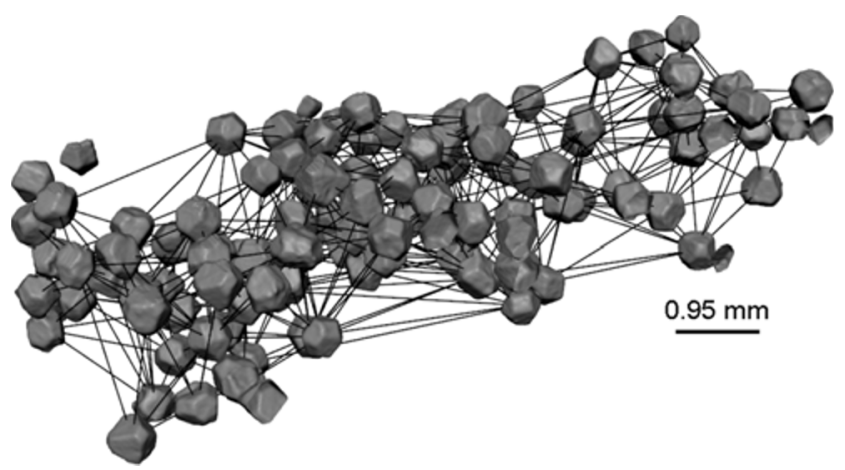

Fig. 4 Connection using the Voronoi diagram, the open meshes are ignored

edge-based and region-based [18]. Since the region-based methods can achieve a better performance when the initial surface is not near the optimal value, in the paper, the method proposed by Lankton and Tannenbaum [19] is used. The evolution of the level set is performed using a localised version of the Chan and Vese model:

$$
\begin{aligned}
\frac{\partial \phi}{\partial t}=\delta_{\epsilon}(\phi)\{ & \frac{w}{k_{1}}\left[\iint_{\Omega} K(\|\boldsymbol{x}-\boldsymbol{y}\|)\left|I(\boldsymbol{x})-f_{1}(\boldsymbol{y})\right|^{2} d \boldsymbol{y}\right. \\
& \left.-\iint_{\Omega} K(\|\boldsymbol{x}-\boldsymbol{y}\|)\left|I(\boldsymbol{x})-f_{2}(\boldsymbol{y})\right|^{2} d \boldsymbol{y}\right] \\
& \left.+\frac{1-w}{k_{2}} \div\left(\frac{\nabla I}{\|\nabla I\|}\right)\right\}
\end{aligned}
$$

where

$$
\begin{aligned}
f_{1}(\boldsymbol{x}) & =\frac{K(\|\boldsymbol{x}-\boldsymbol{y}\|) *\left[H_{\epsilon}(\phi) g(\|\nabla I\|) I(\boldsymbol{x})\right]}{K(\|\boldsymbol{x}-\boldsymbol{y}\|) *\left[H_{\epsilon}(\phi) g(\|\nabla I\|)\right]} \\
f_{2}(\boldsymbol{x}) & =\frac{K(\|\boldsymbol{x}-\boldsymbol{y}\|) *\left[\left(1-H_{\epsilon}(\phi)\right) g(\|\nabla I\|) I(\boldsymbol{x})\right]}{K(\|\boldsymbol{x}-\boldsymbol{y}\|) *\left[\left(1-H_{\epsilon}(\phi)\right) g(\|\nabla I\|)\right]},
\end{aligned}
$$

$\delta_{\epsilon}(x)$ and $H_{\epsilon}(x)$ are the Dirac delta and heaviside functions respectively

$$
\begin{aligned}
\delta_{\epsilon}(x) & =\frac{1}{\pi} \frac{\epsilon}{\epsilon^{2}+x^{2}} \\
H_{\epsilon}(x) & =\frac{1}{2}\left[1+\frac{2}{\pi} \arctan \left(\frac{x}{\epsilon}\right)\right]
\end{aligned}
$$

and $K(\|\boldsymbol{x}-\boldsymbol{y}\|)$ is a function that measures the nonhomogeneity in a neighbourhood of $\boldsymbol{x}$. In this paper, a dense stencil with half width of $r$ is used. A dense stencil includes all the voxels in a cube with edge length equal to $2 r$, centred at the $\boldsymbol{x}$. The volume to mesh conversion was performed using the marching cubes algorithm [20]. Figure 2 shows a slice and the mesh extracted using a global threshold and after applying the region-based algorithm using the same global threshold segmentation as an input. The localised Chan and Vese algorithm allows a better grain segmentation, as there are no artefacts created by the non-homogeneous grey values. A clear difference in the reconstruction of a diamond is highlighted with a red circle in Fig. 2 c and $\mathrm{d}$, in which the mesh is correctly reconstructed using the localised Chan and Vese algorithm, while using the global thresholding there is a hole artefact in the diamond.

\subsection{Diamond abrasive grain extraction}

As two or more abrasive grains may have some faces in common, to correctly extract each grain, a segmentation operation has to be applied. The morphological watershed transform was used [21] to separate each grain. The segmentation procedure can be divided into the following steps:
Fig. 5 Illustration of a segmented diamond grinding wheel and its segments

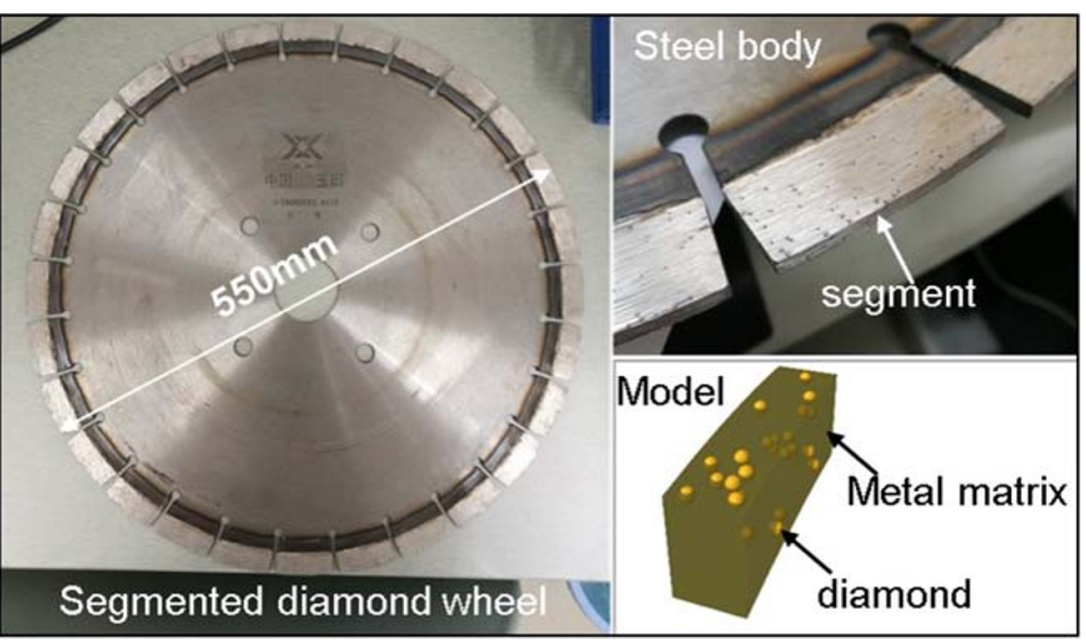


Table 1 XCT parameters used for the measurement of the diamond grinding wheel samples

\begin{tabular}{llll}
\hline $\mathrm{X}$-ray source voltage $(k V)$ & $\mathrm{X}$-ray source target intensity $(\mu \mathrm{A})$ & Mechanical filter, Cu $(\mathrm{mm})$ & Voxel size $(\mu \mathrm{m})$ \\
\hline 175 & 51 & 0.5 & 15.34 \\
\hline
\end{tabular}

1. Compute the local maxima of the signed distance function (the grains are not a portion of the segmented object);

2. Remove all the maxima which difference is less than a threshold to avoid that a grain is split in two or more parts, and this threshold value is set to 1 voxel;

3. Perform the morphological watershed with fully connected option using the local maxima as marker points;

4. Modify the value of the signed distance field to -0.5 voxels if the watershed line intersects a positive value of the distance field;

5. Perform the volume to mesh conversion using the marching cubes algorithm;
6. Remove the mesh of the grinding tool and the small meshes created during the segmentation.

Figure 3 shows the segmentation before and after applying the watershed segmentation. It is evident that it is possible to extract each grain using the proposed method. It should be noted that, during the measurement, it was not possible to distinguish the difference between the grains on the surface of the grinding tool and the air as the material densities of the two are not distinguishable from the measurement. The portion of the grains in contact with the air, therefore, was removed by the algorithm. During the process of finding the surfaces representing the grinding tool, small blobs, as shown in Fig. 3d, which may have

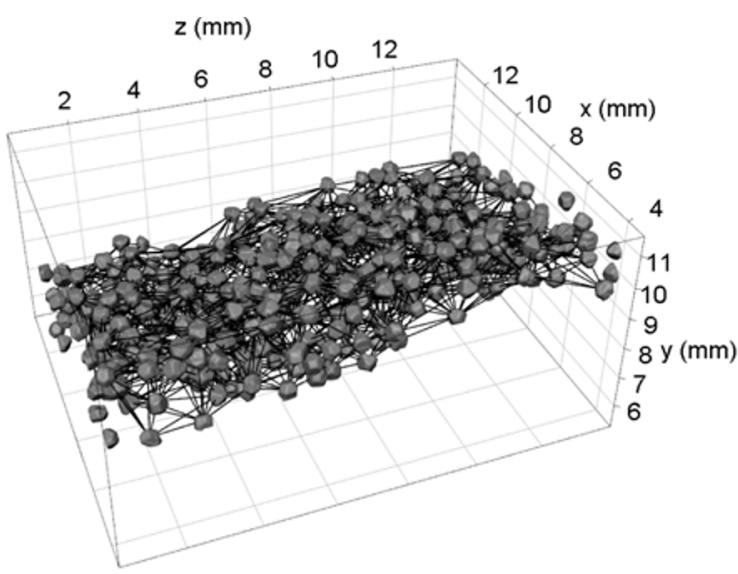

(a) Sample \#1

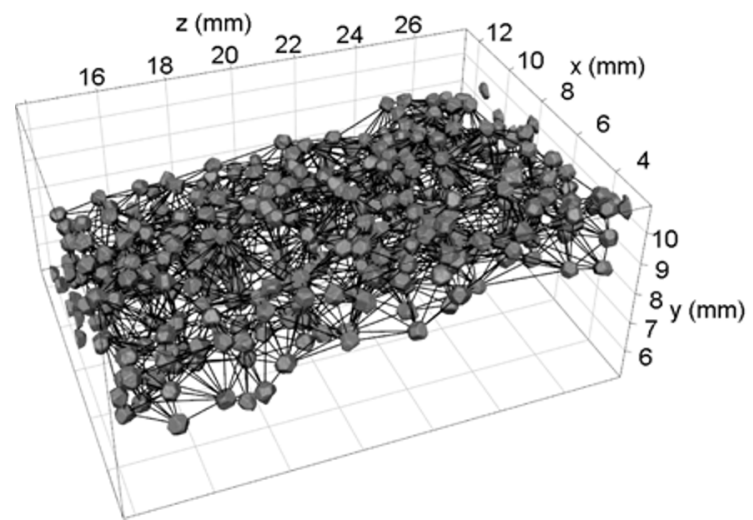

(b) Sample \#2

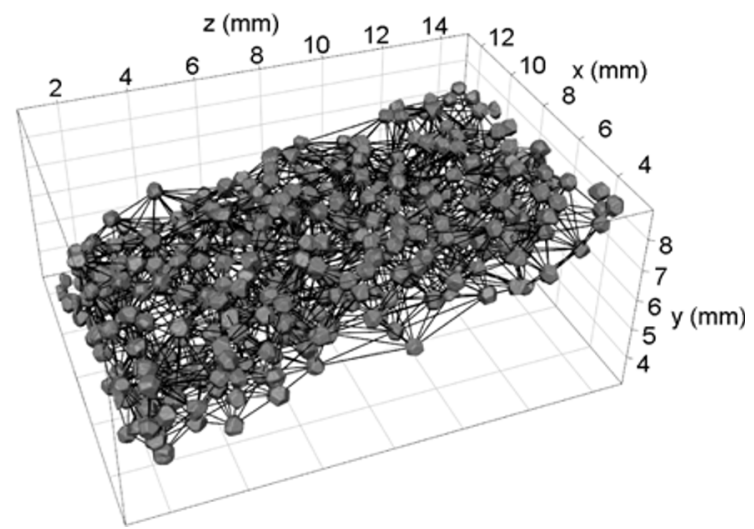

(c) Sample \#3

Fig. 6 Extracted diamonds and computed distribution network 
resulted by measurement noise or smaller (much smaller than the normal grain size) particles in the sample, can be easily discovered and then filtered out. Due to the resolution needed to perform the analysis and the range-to-resolution trade-off of the measurement, sometimes it was not possible to measure a whole grinding wheel, only a portion was analysed. As a result, there are some "open" diamonds with missing portions because they were not in the XCT's measurement volume. As it is not possible to characterise a diamond if a portion is missing, those "open" diamonds were found and then deleted.

\subsection{Network computation}

A functional property of the grinding tool that has to be analysed is the distribution of the grains in the volume. A good uniform distribution of the grains can be measured by computing the variability of the distance from grain to grain. To compute this quantity, a network connecting each centre of mass of the grain has to be constructed. Each mesh was first split into connected components using the $R$ [22] package Rvcg [23]. Each centre of mass can then be computed independently. Since the surface is represented using a triangular mesh, the centre of mass can be computed as

$\boldsymbol{x}_{c m}=\frac{1}{A} \sum_{i=1}^{n_{t r i}} A_{i} \frac{\boldsymbol{v}_{i, 1}+\boldsymbol{v}_{i, 2}+\boldsymbol{v}_{i, 3}}{3}$

where $A=\sum_{i=1}^{n_{t r i}} A_{i}$ is the area of the mesh, $A_{i}$ is the area of the $i$-th triangle and $\boldsymbol{v}_{i, \bullet}$ are its vertices. The connection between the neighbourhood centres of mass

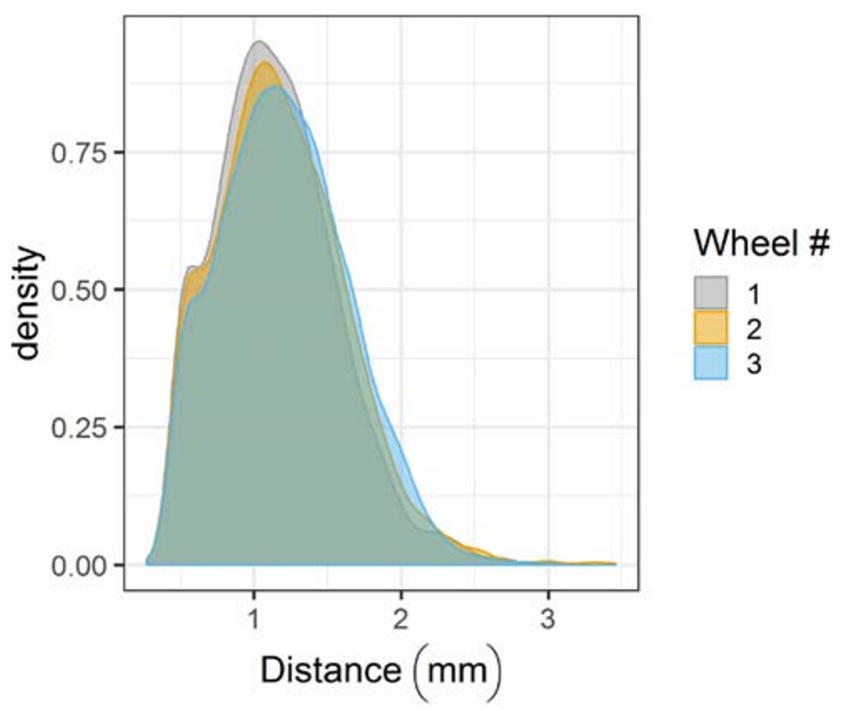

Fig. 7 Distributions of the distances between diamonds
Table 2 Statistics of the centroids' differences

\begin{tabular}{llll}
\hline & \multicolumn{2}{l}{ Sample number } \\
\cline { 2 - 4 } & $\# 1$ & $\# 2$ & $\# 3$ \\
\hline Mean (mm) & 1.15 & 1.19 & 1.21 \\
Standard deviation $(\mathrm{mm})$ & 0.42 & 0.44 & 0.43 \\
\hline
\end{tabular}

can be constructed by connecting the adjacency cells of the Voronoi diagram. Voro++ [24] was used to perform the Voronoi diagram computation. The distance between two grains can then be computed as the distance of two centres of mass. The network of grains from Fig. 3 is shown in Fig. 4, in which the open grains are deleted from the computation because the centre of mass can be affected by the absence of faces.

\section{Quantitative analysis}

In this section, three types of grinding tools are analysed: a set of samples from a segmented diamond grinding wheel, two types of sol-gel balls which will be used to polish different types of marble tiles, and three grinding discs with different 3D controllable abrasive arrangement. In all the test cases, the diamonds have to be segmented first, then a number of indices have to be computed in order to describe the functional properties of the specific grinding tool.

\subsection{Diamond grinding wheel}

As whole diamonds in a grinding wheel can be easily distinguished from the bonding material in the XCT

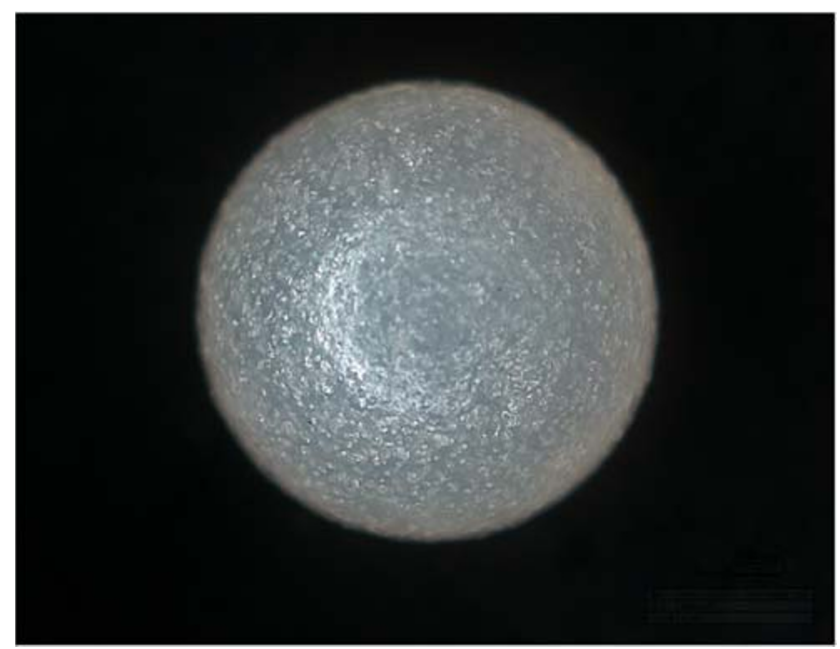

Fig. 8 Three-dimensional microscope image of a sol-gel ball 
Table 3 XCT parameter used for the measurement of the sol-gel balls

\begin{tabular}{lllll}
\hline Nominal diameter & X-ray source voltage $(\mathrm{kV})$ & X-ray source target intensity $(\mu \mathrm{A})$ & Mechanical filter, Cu $(\mathrm{mm})$ & Voxel size $(\mu \mathrm{m})$ \\
\hline $40 \mu \mathrm{m}$ & 152 & 57 & - & 9.89 \\
$80 \mu \mathrm{m}$ & 167 & 59 & 0.1 & 10.58 \\
\hline
\end{tabular}

measured data, the first analysed grinding tool was based on a kind of segmented diamond grinding wheel, as shown in Fig. 5, which was commonly designed for cutting stone and concrete components [25]. Three segment samples designed for a segmented diamond wheel were selected. These samples composed of iron basal matrix and diamond grits distributed inside were fabricated by hot-pressing sintering. The sizes of diamonds range from 425 to $500 \mu \mathrm{m}$. Before sintering, diamonds and matrix powder were mixed by a common rotary mixer. As the density and the distribution of diamonds in every segment may be different, the two factors are the key features to control the wear characteristic of diamond grinding wheel [26].

The XCT measurements for the three samples were carried out using an X-ray power of $175 \mathrm{kV}$ voltage and $51 \mu \mathrm{A}$ of target intensity, with a $0.5-\mathrm{mm} \mathrm{Cu}$ mechanical filter, and voxel size of $15.34 \mu \mathrm{m}$ for the reconstruction, as indicated in Table 1. Figure 6 shows the reconstructed diamonds' meshes and the computed diamonds' distribution network.

A tool to visually check homogeneity of the diamondto-diamond distance between samples is the density plot,
Fig. 9 Reconstructed sol-gel balls with nominal particle of 40 $\mu \mathrm{m}$ and distribution network

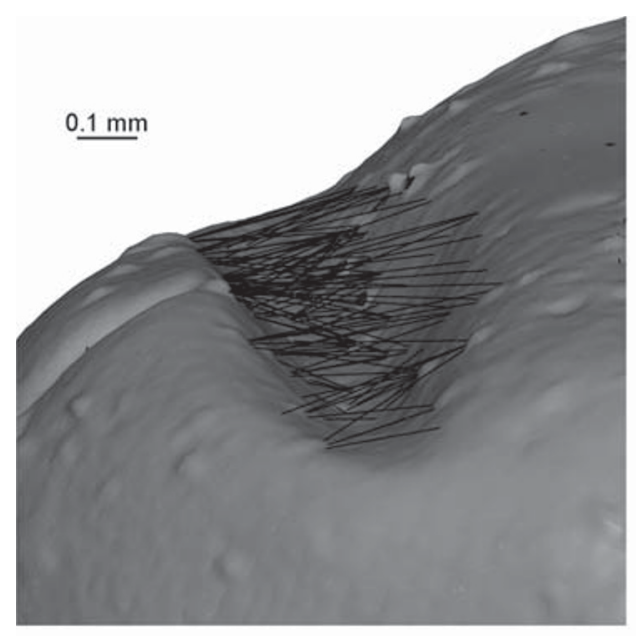

(a) Magnification of Particle \#1 showing the connection between the abrasive grains that intersect the external surface

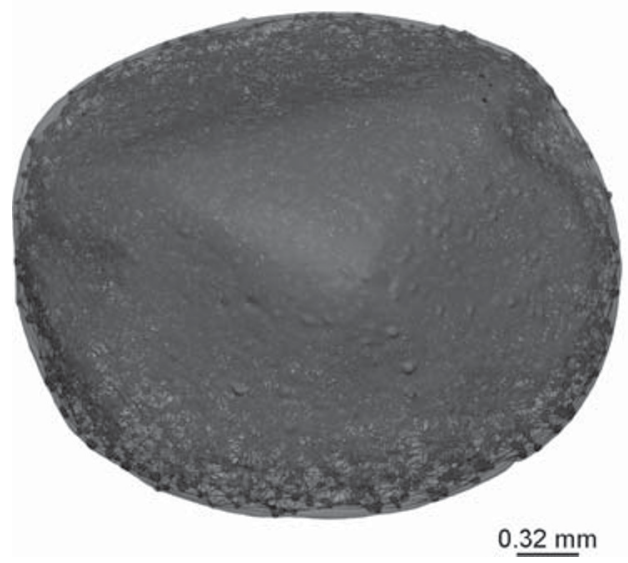

(c) Particle \#2

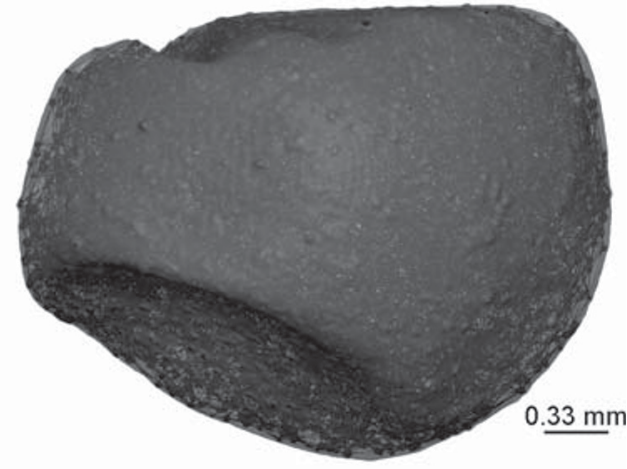

(b) Particle \#1

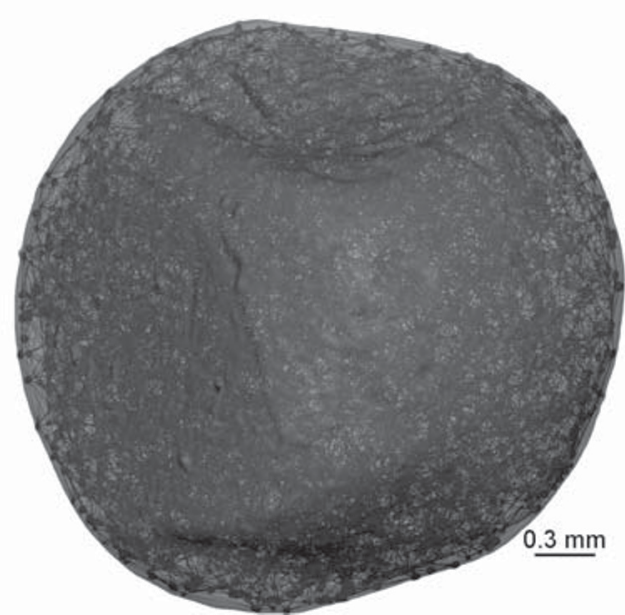

(d) Particle \#3 
Fig. 10 Reconstructed sol-gel balls with nominal particle of 80 $\mu \mathrm{m}$ and distribution network

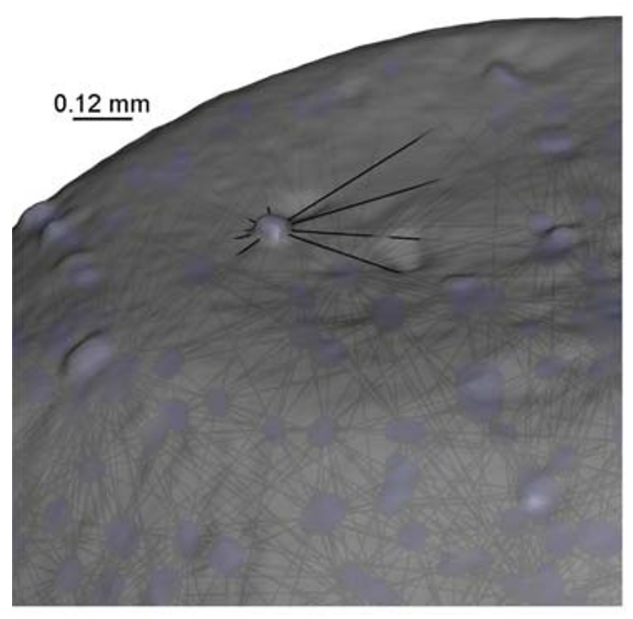

(a) Magnification of Particle \#1

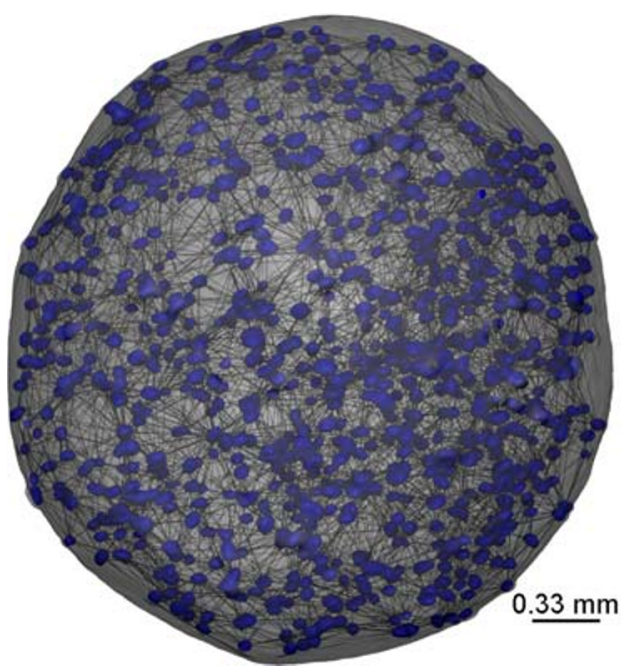

(c) Particle \#2

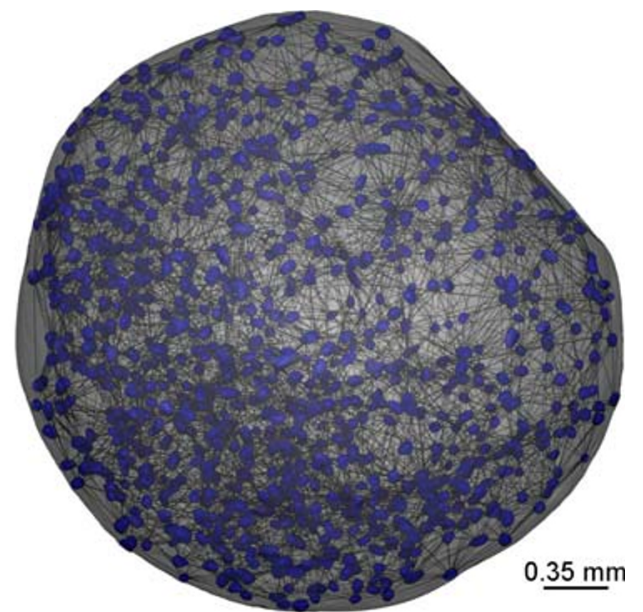

(b) Particle \#1

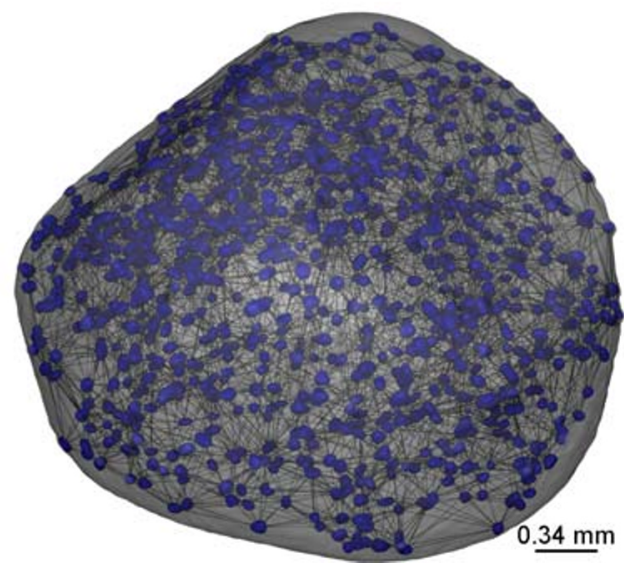

(d) Particle \#3 which is a smoothed version of the histogram computed using a kernel smoothing technique [27]. If there would be a high difference in the shape of the density plot, it would mean that the process has produced grinding tools with heterogeneous diamond-to-diamond distances, i.e. the process is not stable and produces grinding tools with different properties. The density plots of the distances computed between each diamond and its neighbourhood are shown in Fig. 7. The distributions of the distances appear similar between the three samples, i.e. the distribution does not depend on the sample. The mean and the standard deviation, for each sample, are reported in Table 2. Both the means and the standard deviation achieve comparable values. The standard deviations are less than half of the mean distances, which indicates that the dispersion of the diamond grains achieved good spatial uniform distribution with only small deviations. These results indicate that the dispersion of the diamonds inside the three samples was comparable, which provides a quantitative evaluation for assessing a uniform wear of the grinding wheel.

Table 4 Density of the measured sol-gel balls

\begin{tabular}{llll}
\hline Nominal diameter & \multicolumn{2}{l}{ Density (\%) } & \\
\cline { 2 - 4 } & $\# 1$ & $\# 2$ & $\# 3$ \\
\hline $40 \mu \mathrm{m}$ & 1.02 & 0.99 & 0.67 \\
$80 \mu \mathrm{m}$ & 1.13 & 0.80 & 1.08 \\
\hline
\end{tabular}


Fig. 11 Distribution of the distances between the abrasive grains of the two types of sol-gel balls

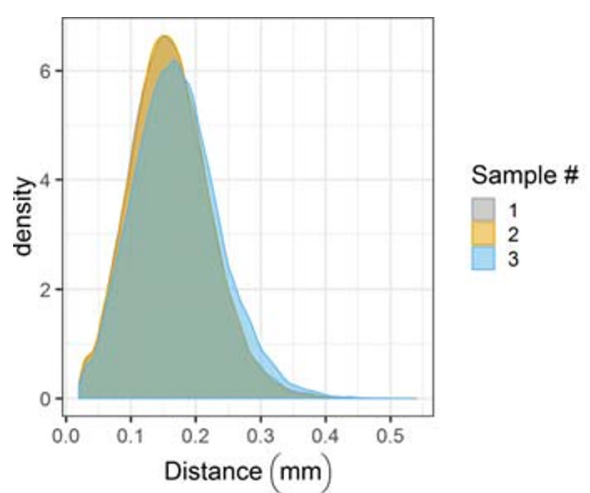

(a) Nominal diameter equal to $40 \mu \mathrm{m}$

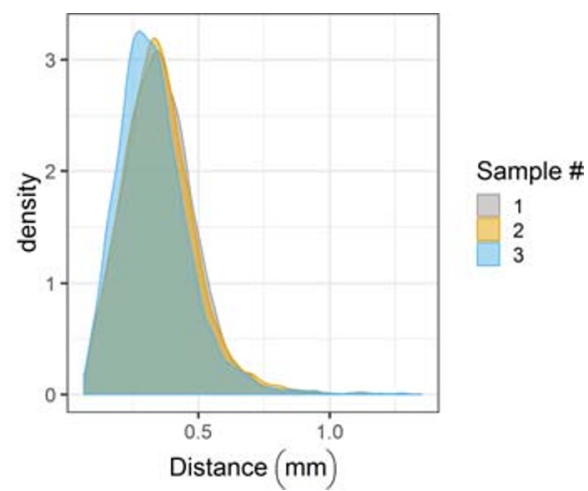

(b) Nominal diameter equal to $80 \mu \mathrm{m}$

\subsection{Sol-gel balls}

In this second test case, two types of sol-gel balls were measured and analysed. The sol-gel ball, as shown in Fig. 8, is a spherical polishing tool which is based on sol-gel technology to polish different types of marble tiles and obtain high-gloss, low surface roughness, and scratch-free surfaces [28]. After a series of preparations include colloid preparation, abrasive dispersion, dripping and solidification, abrasive particles in a sol-gel ball are uniformly dispersed and the size of a sol-gel ball usually range from 2 to $4 \mathrm{~mm}$. In this case, two types of abrasive grains with sizes of $40 \mu \mathrm{m}$ and $80 \mu \mathrm{m}$ were measured using different sets of measurement parameters and voxel sizes as shown in Table 3.

As the two sol-gel balls have different abrasive grain sizes, the grey value of the abrasive grain and the external shell of the sol-gel balls had two different values. It was then possible to perform two reconstructions with different starting values to reconstruct both the surface of the sol-gel ball and the surfaces of the grains. Since the shape of the sol-gel ball is not an exact sphere, it may happen that during the connection of the centres of the Voronoi diagram, some lines intersect the boundary of the ball (see Figs. 9a and 10a). The connections between two centres that intersect the mesh of the reconstructed sol-gel ball were deleted. The reconstructed surfaces are shown in Figs. 9 and 10 for the sol-gel balls with abrasive grains with nominal diameters of $40 \mu \mathrm{m}$ and $80 \mu \mathrm{m}$ respectively.
A first index that can be computed is the percentage of the volume that is occupied by the abrasive grains, in relation to the volume of each sol-gel ball. The percentage of volume is one of the most important indices of sol-gel ball, as the density of grains in a sol-gel ball determines the grinding time required for the grinding process. The density $(\eta)$ can be computed as

$\eta=1-\frac{V_{g}-V_{d i a}}{V_{g}}$

where $V_{g}$ is the volume of the sol-gel ball and $V_{d i a}$ is the volume occupied by the grains. The computed density values are reported in Table 4, which shows that inside both the two types of sol-gel balls there is a similar percentage of volume occupied by the grains, with only sample \#3 of $40 \mu \mathrm{m}$ nominal diameter has a slightly lower volume of grains.

The density plots of the distances of the sol-gel balls are also of importance for the characterisation. The final grain density will be different from the initial abrasive dispersion setting, as the solidification process of the solgel will change the grains' distribution. The density plots of the distances of the analysed sol-gel balls are indicated in Fig. 11. Since it is possible to observe a shift in two of the six analysed samples (sample \#2 of $40 \mu \mathrm{m}$ normal diameter, and sample \#3 of $80 \mu \mathrm{m}$ normal diameter), further statistics were computed: the mean and the standard deviation of the distances. The computed values are reported in Table 5. For each type, one of the three samples has a slightly different

Table 5 Mean and standard deviation of the distances between neighbourhood abrasive grains

\begin{tabular}{|c|c|c|c|c|c|c|}
\hline & \multicolumn{3}{|c|}{ Nominal diameter of $40 \mu \mathrm{m}$} & \multicolumn{3}{|c|}{ Nominal diameter of $80 \mu \mathrm{m}$} \\
\hline & $\# 1$ & $\# 2$ & \#3 & $\# 1$ & $\# 2$ & \#3 \\
\hline Mean $(\mathrm{mm})$ & 0.161 & 0.161 & 0.171 & 0.352 & 0.352 & 0.329 \\
\hline Standard deviation (mm) & 0.061 & 0.060 & 0.066 & 0.133 & 0.140 & 0.140 \\
\hline
\end{tabular}


Fig. 12 Designed pattern of the grinding discs

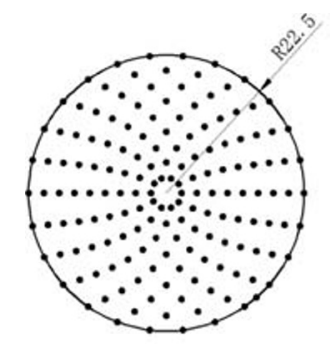

(a) Designed pattern with circular distribution (the first layer)

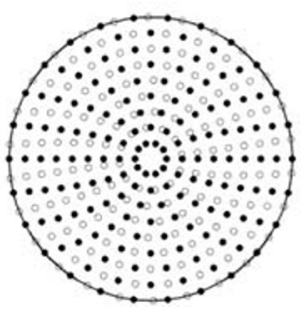

(b) Two abrasive layers (front view)

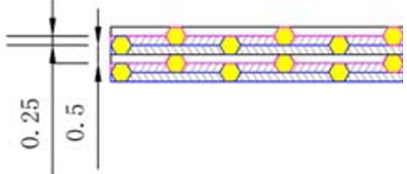

(c) Four abrasive layers (sectional view) mean, i.e. on average the distance of the sample \#3 of the sol-gel balls with nominal diameter of 40 and $80 \mu \mathrm{m}$ is greater by a value of 0.01 and $0.02 \mu \mathrm{m}$ respectively. It should be noted that the dispersion around the mean values (the standard deviations) is comparable, i.e. the variability of the preparation process of the sol-gel balls remains constant on the analysed samples.

\subsection{Grinding discs with designed pattern}

As the design and fabrication of 3D controllable abrasive arrangement wheels have a direct impact on the grinding quality of the workpiece $[29,30]$, in the third test case, three grinding discs with different $3 \mathrm{D}$ controllable abrasive arrangements in the space were measured and analysed. The discs, which were bonded using rein and with a substrate of stainless steel, have an average diamond grain size of $500 \mu \mathrm{m}$. As shown in Fig. 12, the designed pattern forms a circular distribution with a radius of $22.5 \mathrm{~mm}$. There are nine circles distributed along the diameter with the distance of $2.5 \mathrm{~mm}$, and 162 diamonds in a single abrasive layer.

Three grinding discs, as shown in Fig. 13, were then measured and the XCT measurement parameters and the voxel sizes are reported in Table 6.

The reconstructed meshes are shown in Fig. 14. Whenever two diamonds were merged (one was an outlier), they were separated using the watershed transform. The diamonds were then aligned according to their principal axis and manually rotated in order to roughly align the lines of diamonds. A diamond for each grinding wheel (red mesh in the figure) was used to keep track of the upper side of the wheel. The diamonds were initially split in order to have a mesh for each diamond, and then the centres of mass were then computed. Two outliers diamonds of patterns \#1 and \#3 were then manually removed. The next step is to segment each layer of diamonds and analyse them separately. Each layer was then segmented using the nominal pattern: the $x$ $y$ values of the centres of mass were used to draw a regular grid in $x$ and $y$ direction and each cluster was computed (see Fig. 15).

The stability of the process is evaluated by measuring the layer-to-layer distance. To compute the mean difference between each layer, the height values $(z)$ of the centres of mass were fitted using a quadratic model. The model for the first and bottom layer was

$z_{1}(x, y)=\beta_{0}+\beta_{1} x+\beta_{2} y+\beta_{3} x y+\beta_{4} x^{2}+\beta_{5} y^{2}+\epsilon(x, y)$

where $z_{1}(x, y)$ represents the value of the centre of mass, $\beta_{i}$ are coefficients to be estimated and $\epsilon(x, y) \sim \mathcal{N}\left(0, \sigma^{2}\right)$ is an identically and independently distributed normal random error. The linear regression problem was solved using the least squares method [31]. Since the preparation of the grinding wheel is performed layer by layer, the models of
Fig. 13 Three grinding discs with designed pattern

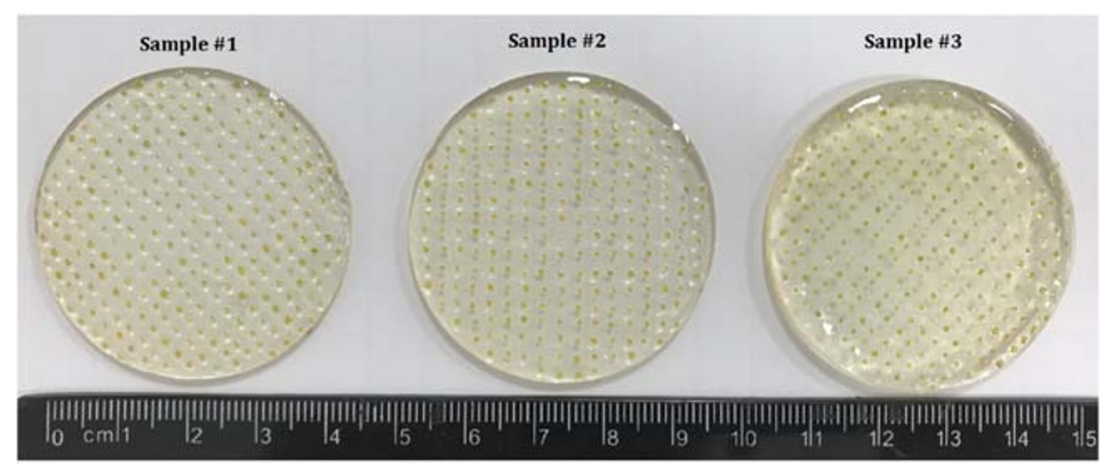


Table 6 XCT parameter used for the measurement of grinding discs

\begin{tabular}{lllll}
\hline Sample number & X-ray source voltage $(k V)$ & X-ray source target intensity $(\mu \mathrm{A})$ & Mechanical filter, Cu $(\mathrm{mm})$ & Voxel size $(\mu$ m) \\
\hline$\# 1$ & 146 & 61 & - & 47.80 \\
$\# 2$ & 147 & 57 & - & 47.91 \\
$\# 3$ & 147 & 57 & - & 48.31 \\
\hline
\end{tabular}

the other layers were designed as the difference between the layer and the previous one

$$
\begin{aligned}
z_{i}(x, y)= & \widehat{z}_{i-1}(x, y)+\beta_{0}+\beta_{1} x+\beta_{2} y \\
& +\beta_{3} x y+\beta_{4} x^{2}+\beta_{5} y^{2}+\epsilon(x, y)
\end{aligned}
$$

where $\widehat{z}_{i}(x, y)$ is the prediction using the model of the $i$-th layer. Figure 16 shows the centres of mass of the layers and the predicted height of each layer.

Table 7 reports the $p$ values of the estimated models. If the $p$ value of a coefficient is less or equal to 0.01 , the corresponding term can be considered statistically different from zero (with a confidence of 0.01). It is possible to observe that, although there is a global parabolic effect, sometimes, there isn't if a layer by layer model is considered. For example, for the pattern \#1, the difference between the second and third layers can be explained only by a constant, that is the goal of the manufacturing process.
After estimating the models, it is possible to compute mean difference between two layers as

$\Delta_{i j}=\frac{1}{A} \iint_{\Sigma} \widehat{z}_{i}(x, y)-\widehat{z}_{j}(x, y) d x d y$

where $\Sigma$ is the domain and $A=\iint_{\Sigma} d x d y$ is its area. Table 8 reports the layer by layer difference. The nominal layer by layer distances were $0.25,0.375$ and $0.5 \mathrm{~mm}$ for the patterns \#1, \#2 and \# 3 respectively. Sample \#1, with an average layer distances of $0.08 \mathrm{~mm}$, shows a $68 \%$ difference with the nominal layer distance of $0.25 \mathrm{~mm}$. Sample \#2, with an average layer distances of $0.2 \mathrm{~mm}$, indicates a $47 \%$ average difference with the nominal layer distance of 0.375 $\mathrm{mm}$. Sample \#3, with an average layer distances of $0.38 \mathrm{~mm}$, presents a $23.4 \%$ difference with the nominal layer distance of $0.5 \mathrm{~mm}$.

\subsection{Discussions}

Three sets of samples with different abrasive grain sizes and bonding materials were selected to validate the

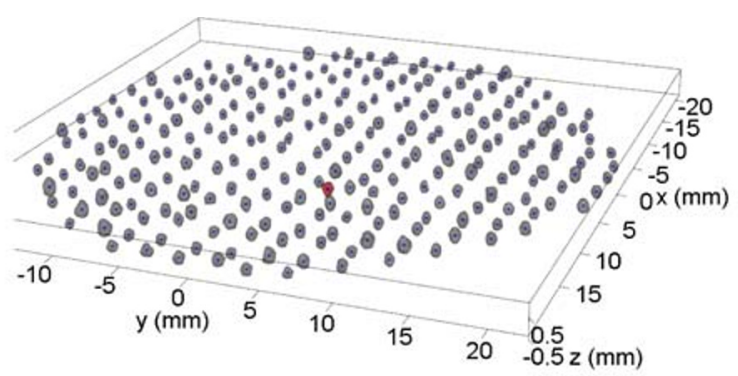

(a) Pattern \#1

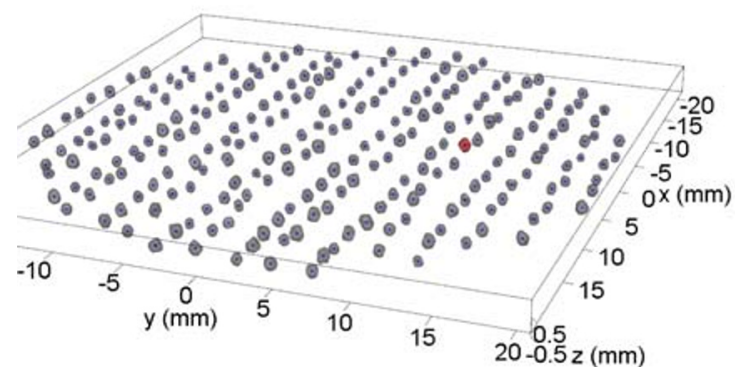

(b) Pattern \#2

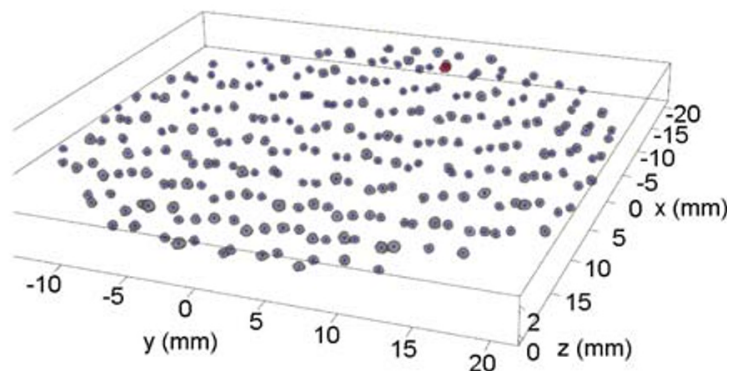

(c) Pattern \#3

Fig. 14 Reconstructed meshes of the grinding discs with three different patterns 


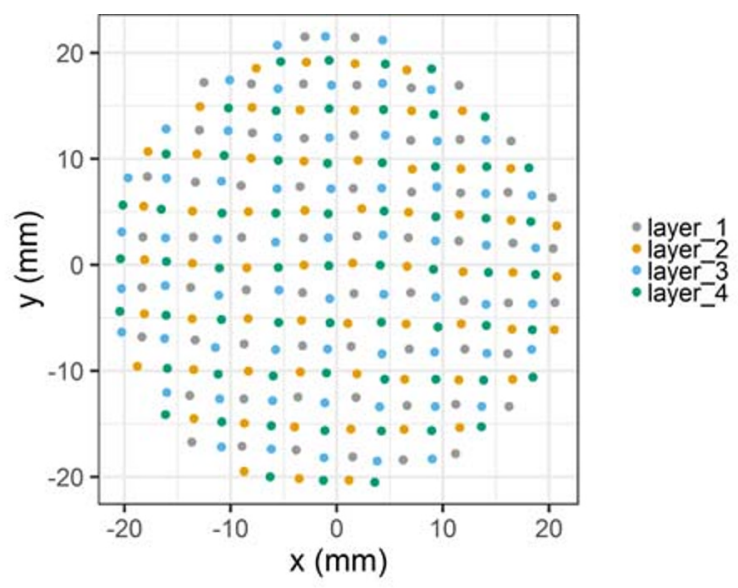

(a) Pattern \#1

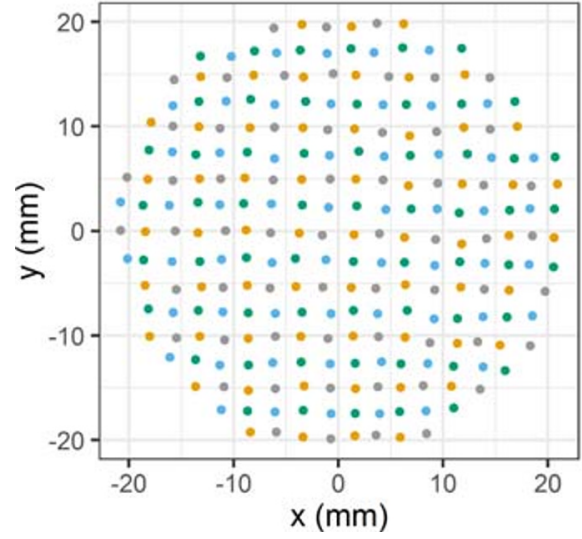

(b) Pattern \#2

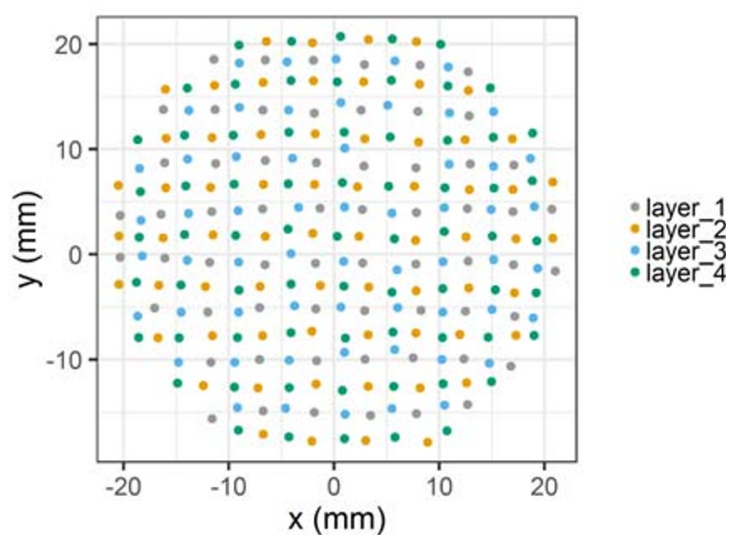

(c) Pattern \#3

Fig. 15 Segmented layers of each pattern

proposed characterisation method. It is clear that the XCT measurement settings differ from three sets of samples. While the settings of X-ray source voltage and target intensity did not indicate any obvious differences, the settings of voxel size varied, where set 1 (diamond grinding wheel) had a voxel size of $15.34 \mu \mathrm{m}$; set 2 (sol-gel ball) had an average voxel size of $10.24 \mu \mathrm{m}$; and set 3 (grinding discs with patterns) of $48.01 \mu \mathrm{m}$. The voxel size is affected by the distance between the sample and the X-ray source, i.e. the closer the sample is located to the $\mathrm{X}$-ray source, the smaller the voxel size. For samples with larger overall sizes, to obtain a full-field measurement, samples such as set 3 had to be located further from the X-ray source, therefore had a relatively larger voxel size (lower resolution). As the three sets have a different range of gain sizes, for example, set 2 has the smallest grains ranging from 40 to $80 \mu \mathrm{m}$, therefore higher resolution was required for set 2 in order to obtain a better segmentation result. The measurement settings for the three sets were also different with the materials that were subject to measure, as X-ray has different penetrate depth upon different materials. For example, as set 1 was bonded with metal matrix, the maximum depth of such grinding wheel that can be measured by the XCT in this paper was much lower than that of set 3 where rein was used as the bonding material.

Each analysed sample set had its own peculiarity during the surface extraction. To extract the surfaces of the diamonds in a grinding tool, a watershed segmentation was applied to separate the diamonds. The diamonds are separated physical objects; therefore, it is possible to correctly separate them via setting an initial threshold value. One seed was set for each diamond to analyse, starting from those points where the watershed segmentation was able to correctly perform the segmentation and the analysis, the mesh was then extracted allowing the analysis of the part. To correctly extract both the diamonds and the external surfaces of the sol-gel balls, two initial threshold values were used to achieve a correct segmentation. Since the materials of the diamonds and the external surface differ, using only one threshold would have resulted a noncorrect segmentation. Using the initial threshold value of the external surface, some of the diamonds may not be selected, due to the different grey values. The used segmentation cannot create new surfaces because a local method was used 


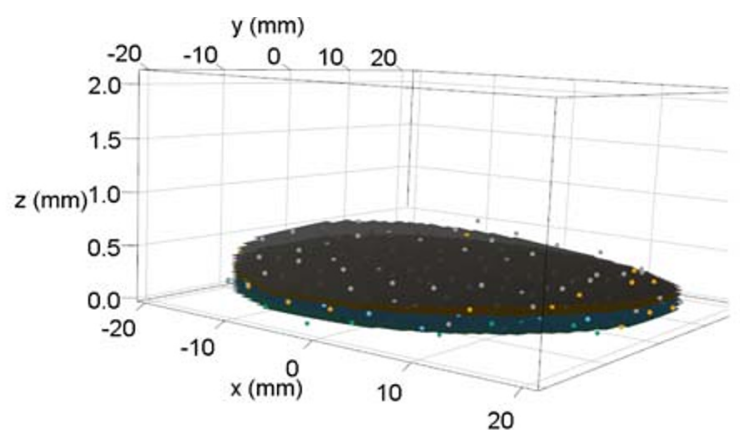

(a) Pattern \#1

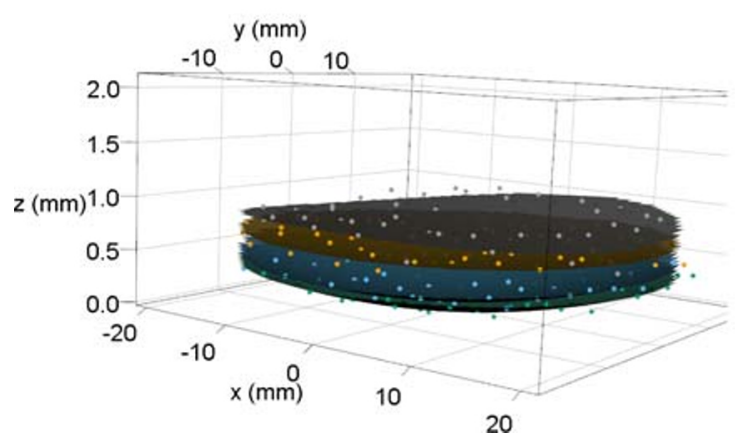

(b) Pattern \#2

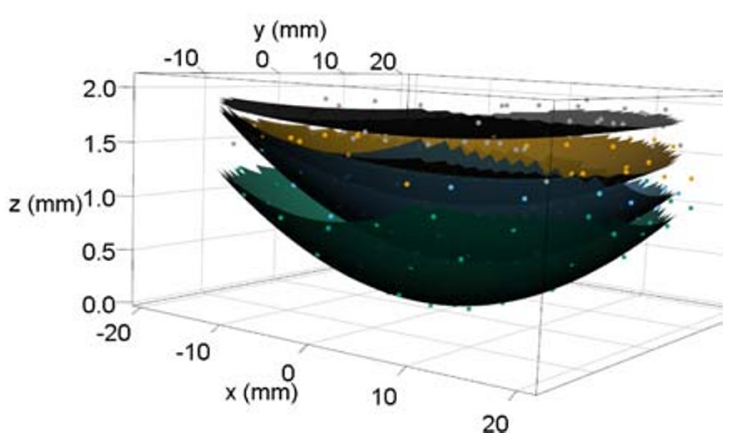

(c) Pattern \#3

Fig. 16 Centres of mass of the reconstructed meshes of the three samples

in the evolution of the level set. Using only one threshold would then lead to a wrong estimation of the density index. In the analysis of set 3, a good alignment allowed a correct classification of the designed layers. Finally, the value of the neighbourhood used in the level set method has to be correctly set if the beam hardening effect is high, and a high width of the windows allows the algorithm to correctly perform the surface segmentation.

\section{Conclusions and future developments}

In this paper, a "true" 3D characterisation of grinding tool using industrial XCT was carried out. Compared to classical methods based on 2.5D surfaces, usually measured with optical/stylus instrumentation, XCT allows to perform a true 3D characterisation with both the surface and internal measurement. Methods that are able to extract the abrasive grains from the XCT volume data were developed and introduced in this paper. To validate the proposed method, three different types of grinding tools specimens were measured and analysed. Each abrasive grain was segmented and the distributions of grains (with both random and designed patterns) were then calculated, plotted and analysed. The quantitative analysis clearly shows the deviations between the measured distribution and the designed pattern of the grinding tool. It can conclude that the industrial XCT-based measurement and characterisation can provide an accurate and comprehensive characterisation of the grinding tool, therefore further in assist with

Table $7 p$ values of the estimated models

\begin{tabular}{|c|c|c|c|c|c|c|c|c|c|c|c|c|}
\hline \multirow[b]{2}{*}{$\beta_{0}$} & \multicolumn{4}{|c|}{ Pattern \#1 } & \multicolumn{4}{|c|}{ Pattern \#2 } & \multicolumn{4}{|c|}{ Pattern \#3 } \\
\hline & $l_{1}$ & $l_{2}-l_{1}$ & $l_{3}-l_{2}$ & $l_{4}-l_{3}$ & $l_{1}$ & $l_{2}-l_{1}$ & $l_{3}-l_{2}$ & $l_{4}-l_{3}$ & $l_{1}$ & $l_{2}-l_{1}$ & $l_{3}-l_{2}$ & $l_{4}-l_{3}$ \\
\hline$\beta_{1}$ & $<0.01$ & $<0.01$ & $<0.01$ & $<0.01$ & $<0.01$ & $<0.01$ & $<0.01$ & $<0.01$ & $<0.01$ & $<0.01$ & $<0.01$ & $<0.01$ \\
\hline$\beta_{2}$ & 0.8 & 0.07 & 0.68 & 0.01 & $<0.01$ & 0.24 & 0.13 & 0.36 & 0.04 & $<0.01$ & $<0.01$ & $<0.01$ \\
\hline$\beta_{3}$ & $<0.01$ & $<0.01$ & 0.38 & 0.79 & $<0.01$ & $<0.01$ & $<0.01$ & 0.28 & 0.08 & $<0.01$ & $<0.01$ & 0.06 \\
\hline$\beta_{4}$ & 0.09 & 0.38 & 0.22 & 0.08 & $<0.01$ & 0.86 & 0.26 & 0.47 & 0.48 & 0.04 & $<0.01$ & 0.22 \\
\hline$\beta_{5}$ & $<0.01$ & $<0.01$ & 0.21 & 0.79 & $<0.01$ & 0.08 & $<0.01$ & $<0.01$ & $<0.01$ & $<0.01$ & $<0.01$ & $<0.01$ \\
\hline$\beta_{6}$ & $<0.01$ & $<0.01$ & 0.81 & 0.05 & $<0.01$ & 0.56 & $<0.01$ & 0.18 & $<0.01$ & 0.04 & $<0.01$ & $<0.01$ \\
\hline
\end{tabular}


Table 8 Layer by layer mean difference

\begin{tabular}{llll}
\hline & $l_{2}-l_{1}(\mathrm{~mm})$ & $l_{3}-l_{2}(\mathrm{~mm})$ & $l_{4}-l_{3}(\mathrm{~mm})$ \\
\hline Sample \#1 & 0.07 & 0.08 & 0.09 \\
Sample \#2 & 0.12 & 0.26 & 0.22 \\
Sample \#3 & 0.42 & 0.32 & 0.41 \\
\hline
\end{tabular}

controlling the manufacturing process of grinding tool, and in predict its performance. As the aim of the proposed work focused on using industrial XCT measurement for grinding tool characterisation, the accuracy and precision of the XCT measurement $[32,33]$ will be studied in our upcoming research work.

Funding This study was financially supported by the Institute of Manufacturing Engineering of Huaqiao University. JL, HH, GH, CC and XX were supported by the National Nature Science Foundation of China (Ref: 51835004) and Xiamen Science and Technology Project (3502Z20183019); QQ was supported by the UK's Engineering and Physical Sciences Research Council (EPSRC) funding of the EPSRC UKRI Innovation Fellowship (Ref: EP/S001328/1); LP was supported by the EPSRC Fellowship in Manufacturing (Ref: EP/R024162/1).

Open Access This article is licensed under a Creative Commons Attribution 4.0 International License, which permits use, sharing, adaptation, distribution and reproduction in any medium or format, as long as you give appropriate credit to the original author(s) and the source, provide a link to the Creative Commons licence, and indicate if changes were made. The images or other third party material in this article are included in the article's Creative Commons licence, unless indicated otherwise in a credit line to the material. If material is not included in the article's Creative Commons licence and your intended use is not permitted by statutory regulation or exceeds the permitted use, you will need to obtain permission directly from the copyright holder. To view a copy of this licence, visit http:// creativecommonshorg/licenses/by/4.0/.

\section{References}

1. Brinksmeier E, Werner F (1992) Monitoring of grinding wheel wear. CIRP Annals 41(1):373-376

2. Zuo H, Koshino K, Suzuki S (2007) An effective noise removal technique for recovering the shapes of diamond abrasive grains in slm images degraded by clustered spike noise. Optik-International Journal for Light and Electron Optics 118(4):187-194

3. Qiao G, Dong G, Zhou M (2013) Simulation and assessment of diamond mill grinding wheel topography. Int J Adv Manuf Technol 68(9-12):2085-2093

4. Zhang X Fundamental research on the detection technology of grinding wheel topography based on binocular vision principle, Nanjing: Nanjing University of Aeronautics and Astronautics

5. Xie J, Wei F, Zheng J, Tamaki J, Kubo A (2011) 3D laser investigation on micron-scale grain protrusion topography of truncated diamond grinding wheel for precision grinding performance. Int J Mach Tools Manuf 51(5):411-419

6. Cui C, Xu X, Huang H, Hu J, Ye R, Zhou L, Huang C (2013) Extraction of the grains topography from grinding wheels. Measurement 46(1):484-490
7. Xie J, Lu Y (2011) Study on axial-feed mirror finish grinding of hard and brittle materials in relation to micron-scale grain protrusion parameters. Int J Mach Tools Manuf 51(1):84-93

8. Ye R, Jiang X, Blunt L, Cui C, Yu Q (2016) The application of 3D-motif analysis to characterize diamond grinding wheel topography. Measurement 77:73-79

9. Huo F, Jin Z, Kang R, Guo D, Yang C (2008) Recognition of diamond grains on surface of fine diamond grinding wheel. Frontiers of Mechanical Engineering in China 3(3):325-331

10. Kruth JP, Bartscher M, Carmignato S, Schmitt R, De Chiffre L, Weckenmann A (2011) Computed tomography for dimensional metrology. CIRP Annals 60(2):821-842

11. Müller P, Cantatore A, Andreasen JL, Hiller J, De Chiffre L (2013) Computed tomography as a tool for tolerance verification of industrial parts. Procedia CIRP 10:125-132

12. Ziółkowski G, Chlebus E, Szymczyk P, Kurzac J (2014) Application of X-ray CT method for discontinuity and porosity detection in 316L stainless steel parts produced with SLM technology. Arch Civ Mech Eng 14(4):608-614

13. Hanke R, Fuchs T, Uhlmann N (2008) X-ray based methods for non-destructive testing and material characterization. Nuclear Instruments and Methods in Physics Research Section A: accelerators, Spectrometers, Detectors and Associated Equipment 591(1):14-18

14. Nikon Metrology, XT H 225. https://www.nikonmetrology.com/ en-gb/product/xt-h-225

15. Nikon Metrology NV, Nikon CT-Pro (2016)

16. Ibanez L, Schroeder W, Ng L, Cates J (2003) The ITK software guide, Kitware, Inc. 1st Edition

17. Mirebeau J-M, Fehrenbach J, Risser L, Tobji S Anisotropic diffusion in ITK. arXiv: 1503.00992

18. Soomro S, Munir A, Choi KN (2018) Hybrid two-stage active contour method with region and edge information for intensity inhomogeneous image segmentation. PloS One 13(1):1-20

19. Lankton S, Tannenbaum A (2008) Localizing region-based active contours. IEEE Trans Image Process 17(11):2029-2039. https://doi.org/10.1109/TIP.2008.2004611

20. Lorensen WE, Cline HE (1987) Marching cubes: a high resolution 3D surface construction algorithm. ACM Computer Graphics 21(4):163-169

21. Beare R, Lehmann G (2006) The watershed transform in ITKdiscussion and new developments. The Insight Journal 1:124

22. R Core Team (2018) R: a language and environment for statistical computing, R Foundation for Statistical Computing, Vienna, Austria. https://www.R-project.org/

23. Schlager S (2017) Morpho and Rvcg - shape analysis in R. In: Zheng G, Li S, Szekely G (eds) Statistical shape and deformation analysis. Academic Press, pp 217-256

24. Rycroft C (2009) Voro++: a three-dimensional Voronoi cell library in $\mathrm{C}++$, Tech. rep., Lawrence Berkeley National lab.(LBNL), Berkeley, CA (United States)

25. Huang G, Zhang M, Huang H, Guo H, Xu X (2018) Estimation of power consumption in the circular sawing of stone based on tangential force distribution. Rock Mech Rock Eng 51(4):1249_ 1261

26. Xu X, Li Y, Zeng W, Li L (2002) Quantitative analysis of the loads acting on the abrasive grits in the diamond sawing of granites. $J$ Mater process Technol 129(1-3):50-55

27. Peter H, Kernel D (1985) Estimation of a distribution function. Communications in Statistics-Theory and Methods 14(3):605-620

28. Huang S, Lu J, Chen S, Huang H, Xu X, Cui C (2019) Study on the surface quality of marble tiles polished with sol-gel derived pads. J Sol-Gel Sci Technol, pp 1-11 
29. Qiu Y, Huang H, Xu X (2018) Effect of additive particles on the performance of ultraviolet-cured resin-bond grinding wheels fabricated using additive manufacturing technology. Int $\mathbf{J}$ Adv Manuf Technol 97(9-12):3873-3882

30. Qiu Y, Huang H (2019) Research on the fabrication and grinding performance of 3-dimensional controllable abrasive arrangement wheels. The International Journal of Advanced Manufacturing Technology, pp 1-15

31. Johnson RA, Wichern DW et al (2002) Applied multivariate statistical analysis, vol 5. Prentice hall, Upper Saddle River
32. Müller P, Hiller J, Dai Y, Andreasen J, Hansen HN, De Chiffre L (2014) Estimation of measurement uncertainties in X-ray computed tomography metrology using the substitution method. CIRP J Manuf Sci Technol 7(3):222-232

33. Villarraga-Gómez H, Thousand JD, Smith ST Empirical approaches to uncertainty analysis of x-ray computed tomography measurements: a review with examples, Precision Engineering

Publisher's note Springer Nature remains neutral with regard to jurisdictional claims in published maps and institutional affiliations.

\title{
Affiliations
}

Luca Pagani $^{1}$ - Qunfen $\mathrm{Qi}^{1}$ (D) . Jing $\mathrm{Lu}^{2}$ - Hui Huang ${ }^{2}$ - Guoqin Huang ${ }^{2}$. Changcai Cui ${ }^{2}$ Xipeng $\mathrm{Xu}^{2}$. Xiangqian Jiang ${ }^{1}$

\author{
Luca Pagani \\ 1.pagani@hud.ac.uk \\ Jing $\mathrm{Lu}$ \\ lujing26@hqu.edu.cn \\ Hui Huang \\ huangh@hqu.edu.cn \\ Guoqin Huang \\ smarthgq@hqu.edu.cn \\ Changcai Cui \\ cuichc@hqu.edu.cn
}

Xipeng Xu

xpxu@hqu.edu.cn

Xiangqian Jiang

x.jiang@hud.ac.uk

1 EPSRC Future Advanced Metrology Hub, School of Computing and Engineering, University of Huddersfield, Huddersfield, HD1 3DH, UK

2 Institute of Manufacturing Engineering, Huaqiao University, Xiamen, 361021, Fujian, People's Republic of China 